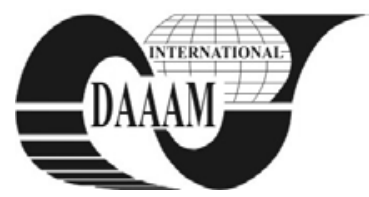

Annals of DAAAM for 2011 \& Proceedings of the 22nd International DAAAM Symposium, Volume 22, No. 1, ISSN 1726-9679 ISBN 978-3-901509-83-4, Editor B. Katalinic, Published by DAAAM International, Vienna, Austria, EU, 2011 Make Harmony between Technology and Nature, and Your Mind will Fly Free as a Bird Annals \& Proceedings of DAAAM International 2011

\title{
POSSIBILITY OF SENSORLESS CONTROL FOR IPMSM IN LOW SPEED RANGE
}

\author{
VESELY, L[ibor] \& POHL, L[ukas]
}

\begin{abstract}
This paper describes a possibility for estimation of the rotor position in low speed of interior permanent synchronous motor (IPMSM) The IPMSM has salient rotor, this means the stator inductance changes depending on the current position of the rotor, so information about rotor position is contained in changes of stator inductance. Stator inductance changes are identified by the injected high frequency signal Key words: sensorless control, IPMSM, estimation, injected signal
\end{abstract}

\section{INTRODUCTION}

Permanent magnet synchronous motors (PMSM) have been used in many industrial applications because they have several inherent advantages e.g. rugged construction, easy maintenance, high power factor and suitability for wide speed ranges of constant power operation. However, rotor position information is necessary even to be able to control drive speed. Conventional speed and position sensing was obtained using encoder, resolver. These sensors add cost, weight. Therefore algorithms for rotor position and speed estimation from electrical quantities have been developed by many authors. These algorithms are often based on state estimation idea (e.g. extended Kalman filter, nonlinear observer). However many of developed algorithms provide bad performance in the low speed region. Reason is obvious. The back electromotive force (EMF) is too small in the low speed, which is crucial to estimate. Small back EMF, noise measurement and inaccurate model of PMSM are the main reason of bad performance.

This paper describes algorithm of rotor position estimation, which is based on identification of stator inductance. PMSM can be classified by rotor construction into surface permanent magnet synchronous motor (SPMSM) and interior permanent synchronous motor (IPMSM). The IPMSM has salient rotor, this means the stator inductance changes depending on the current position of the rotor, so information about rotor position is contained in changes of stator inductance. Stator inductance changes are identified by the injected high frequency signal $(100-400 \mathrm{~Hz})$.
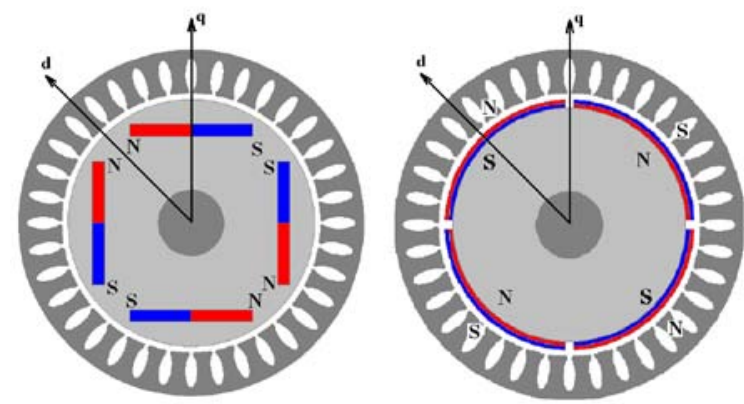

Fig. 1. a)IPMSM $\left(L_{d} \neq L_{q}\right)$

b) SPMSM ( $\left.\mathrm{L}_{\mathrm{d}}=\mathrm{L}_{\mathrm{q}}\right)$

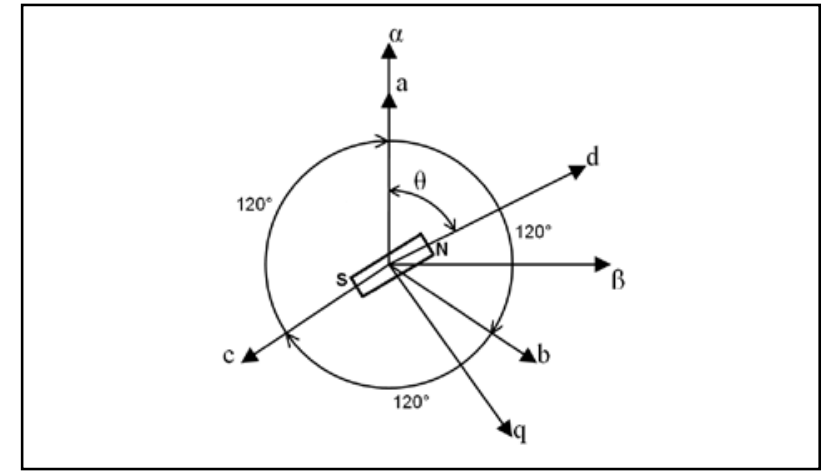

Fig. 2. Model of IPMSM

Fig. 1. a) shows interior permanent magnet motor with salient rotor type. Fig. 1. b) shows surface permanent magnet motor with non-salient rotor type.

\section{MODEL OF IPMSM}

The model of IPMSM is derived from schematic representation of stator and rotor windings. The three-phase windings are representation by phase a, b, c.

Figure 2 shows the model of the PMSM. The d-q frame shows the synchronously rotating reference frame where the $d$ axis coincides with the north pole of the rotor. The orthogonal two-phase $\alpha-\beta$ frame is fixed to the stator windings and $\theta$ represents the actual angle of the rotor position (Bowen et at., 2001).

The following assumptions are made in the derivation:

- The induced EMF is sinusoidal

- Eddy currents and hysteresis losses are negligible

- There is no cage on the rotor

The two axis form of the equations (1) and (2) can be obtained with these assumptions above (Rahman \& Hiti, 2003)

$$
\begin{aligned}
& u_{d}=R i_{d}+\frac{d \Psi_{d}}{d t}-\omega_{s} \Psi_{q} \\
& u_{q}=R i_{q}+\frac{d \Psi_{q}}{d t}+\omega_{s} \Psi_{d}
\end{aligned}
$$

where

$$
\begin{gathered}
\Psi_{d}=L_{d} i_{d}+\Psi_{P M} \\
\Psi_{q}=L_{q} i_{q},
\end{gathered}
$$

$u_{d}, u_{q}$ are the $d, q$ axis voltages, $i_{d}, i_{q}$ are the $d, q$ axis stator currents, $L_{d}, L_{q}$ are the $d, q$ axis inductances , $\Psi_{d}, \Psi_{q}$ are the $d, q$ axis stator flux linkages, $R$ is stator resistance and $\omega_{s}$ is synchronous frequency. These equations directly follow form the equations of the smooth-air-gap synchronous machine expressed in the rotor reference frame, but instead of the flux linkage produced by field winding, now the magnetic flux $\Psi_{P M}$ is present 


\section{IDENTIFICATION OF STATOR INDUCTANCE}

Estimation of rotor position at low speed is the most complex. It can not be used the same principles as in estimating the initial rotor position. Identification of stator inductance with high frequency signal must be done simultaneously with the vector control of synchronous machine. The algorithm of identification of stator inductance must be sufficiently fast.

The equations (5) and (6) describing the stator inductance in the $\alpha-\beta$ frame (Vas, 1998).

$$
\begin{gathered}
L_{\alpha}=L_{0}+L_{1} \cos (2 \theta) \\
L_{\beta}=L_{0}-L_{1} \cos (2 \theta)
\end{gathered}
$$

where

$$
\begin{aligned}
& L_{0}=\frac{L_{d}+L_{q}}{2} \\
& L_{1}=\frac{L_{d}-L_{q}}{2}
\end{aligned}
$$

$L_{\alpha}, L_{\beta}$ are the $\alpha, \beta$ axis inductances and $\theta$ is electrical rotor position.

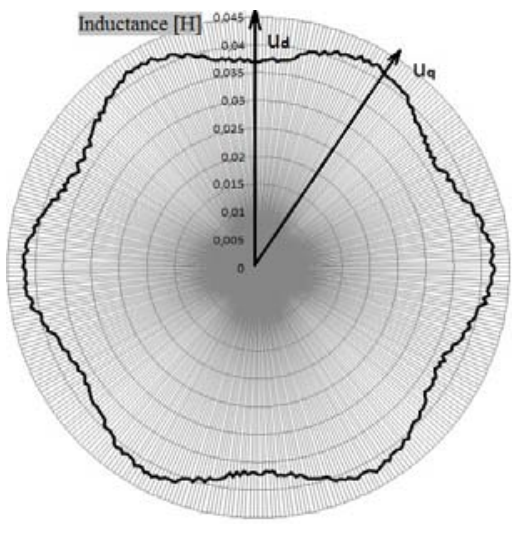

Fig. 3. Changes of stator inductance

\section{RESULTS}

We must solve two basic questions, so as we could use the method of high frequency signal. How we will inject a high frequency signal to tha control loop and how this signal will be processed. (Sungmin et al., 2010)(Jeong et at., 2003)

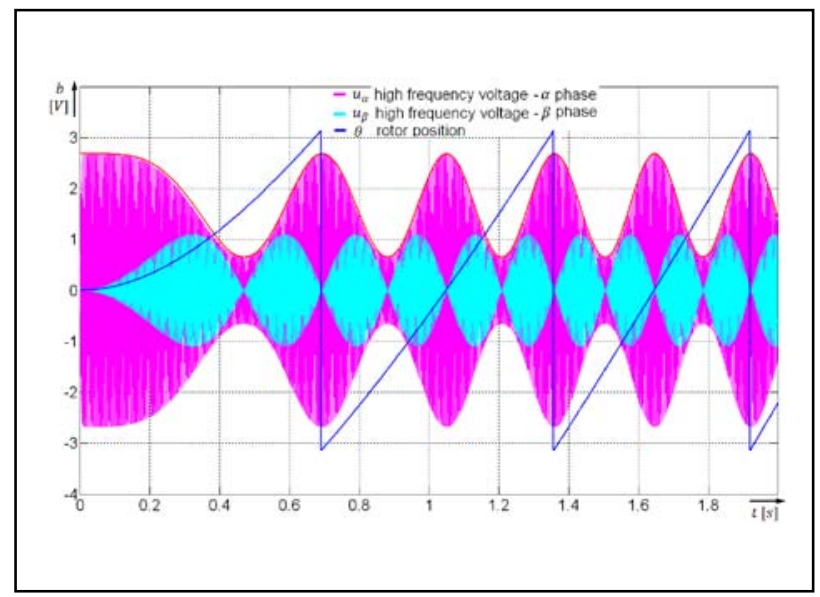

Fig. 4. Amplitude of high frequency voltage

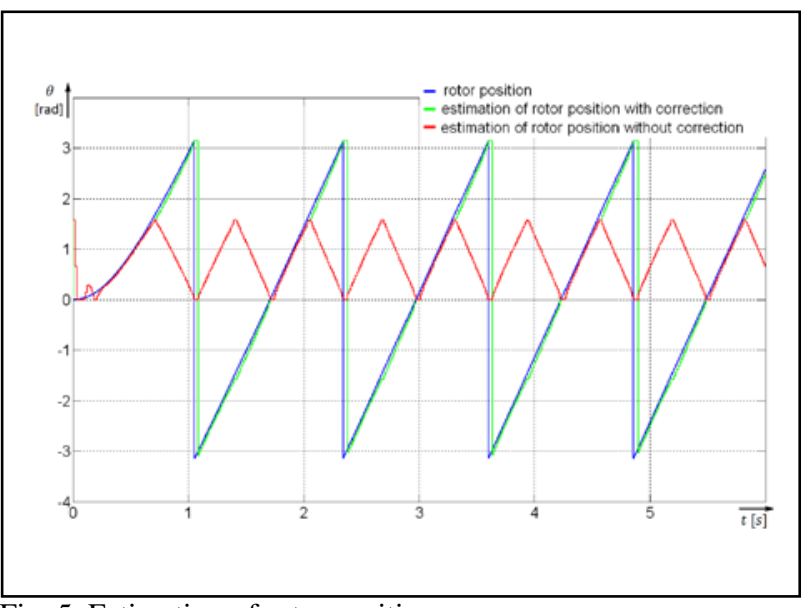

Fig. 5. Estimation of rotor position

High frequency testing signal are released into the $\alpha$ axis, after passing through the engine, its amplitude is captured in Figure 4. Correlation method determined the amplitude of the test signal and subsequent calculation estimated the rotor position.

Figure 5 shows time delay between actual and estimated rotor position, which is caused by time integration. The greater the integration period for the calculation of correlation, it gives greater resistance to noise.

\section{CONCLUSION}

This algorithm provide good performance in the low speed region, but in calculating the estimated position is used the principle of correlation, so this method is not suitable for medium and high speed. The correlation ensures the separation of high frequency signal from motor control signal and increase resistance to noise. Thus, this algorithm adds sensorless control algorithms based on the observation of back emf.

My future work will focus on the implementation of this algorithm in IN cRIO system working in real-time.

\section{ACKNOWLEDGEMENTS}

The research has been supported by Czech Science Foundation under the project GA P103/10/0647 "Intelligent Electrical Drives Predictive and Robust Control Algorithms" and the Ministry of Education of the Czech Republic under the project 1M0567 "Center for Applied Cybernetics" and by Freescale Semiconductor Czech Republic (former division of Motorola).

\section{REFERENCES}

Bowen, C.; Jihua, Z. \& Zhang, R. (2001). Modeling and Simulation of Permanent Magnet Synchronous, Proceedings of the Fifth International Conference on Electrical Machines ans Systems pp. 905-908

Jeong, Y.; Lorenz, R. D.; Jahns, T. M. \& Sul, S. (2003). Initial Rotor Position Estimation of an Interior Permanent Magnet Synchronous Machine Using Carrier-Frequency Injection Methods, 0-7803-7817-2, pp.1218-1223

Rahman, M. K. \& Hiti, S. (2003). Identification of Machine Parametres of a Sychronous Motor, 0-7803-7883-0/03, Torrance,USA

Sungmin, K.; Young-Doo, Y.; Seung-Ki, S.; Kozo, I. \& Koji, T. (2010). Parameter Independent Maximum Torque per Ampere (MTPA) Control of IPM Machine Based on Signal Injection, APEC, Feb 2010, ISSN: 1048-2334

Vas, P. (1998). Sensorless vector and direct torque control, ISBN 0-19-856465-1, Oxford 